\title{
REBOA: método adyuvante o puente en el control de la hemorragia
}

\section{REBOA: Adjuvant or bridge in the control of bleeding}

\author{
Roberto Castañeda-Gaxiola ${ }^{1}$, Luis J. Fernández-López ${ }^{2 *}$, Edmundo Arias-Gómez ${ }^{3}$, Paola Prieto-Olivares ${ }^{4}$, \\ y Manuel E. Marquina-Ramírez ${ }^{1}$ \\ ${ }^{1}$ Cirugía Vascular periférico, Centro Médico ABC Santa Fe; ${ }^{2}$ Cirugía General, Centro Médico ABC Santa Fe; ${ }^{3}$ Cirugía General, Hospital Ángeles \\ Pedregal; ${ }^{4}$ Cirugía General, Trasplantes, Hospital Ángeles Pedregal. Ciudad de México, México
}

\begin{abstract}
Resumen
Introducción: La oclusión endovascular con balón de la aorta para reanimación (REBOA) es un procedimiento endovascular que consiste en insuflar un balón distensible en la aorta a través de un acceso arterial femoral con el objetivo de mejorar la perfusión de órganos vitales. La base teórica es similar a la de la toracotomía de reanimación (TR) con pinzamiento aórtico o masaje cardíaco abierto, pero con menor invasividad y morbilidad. La aorta se divide en tres zonas, considerados el diafragma y las arterias renales; en la utilización de la REBOA se prefiere siempre insuflar en la zona 1 sin importar cuál sea la fuente de la hemorragia y sólo en casos específicos se utiliza en la zona 3. La REBOA se emplea en múltiples casos de choque hemorrágico, secundario o no a traumatismo, y en paro cardíaco con buenos resultados, como lo muestran múltiples estudios en pacientes seleccionados; sin embargo, con estos estudios no pueden dilucidarse sus limitantes o alcances. Conclusiones: La aplicación de REBOA debe determinarse en cada escenario con base en el entrenamiento, experiencia, recursos locales y tiempos de evacuación.
\end{abstract}

Palabras clave: Procedimientos endovasculares. Ruptura aórtica. REBOA. Traumatismo múltiple. Hemorragia. Abdomen.

\section{Abstract}

Background: Resuscitative endovascular balloon occlusion of the aorta (REBOA) is an endovascular procedure that consists of inflating a compliant balloon in the aorta through a femoral arterial access, with the intention of improving vital organs perfusion. Theoretical basis is similar to resuscitative thoracotomy (RT), with aortic clamping and/or open heart massage, but less invasive and with a lower morbidity. The aorta is divided into 3 zones considering the diaphragm and renal arteries, when using REBOA it is always preferred to inflate the balloon in Zone 1 regardless of the origin of the bleeding and it is only used in Zone 3 in exceptional cases. REBOA is used in various cases of hemorrhagic shock, either secondary to traumatismo or not, it is also used in cardiac arrest cases with good results, as shown in multiple studies in selected patients, nevertheless, using these studies the limitations or the scope of the procedure can not be clarified. Conclusions: The implementation $R E B O A$ should be determined based on training, experience, local resources and evacuation times.

Key words: Endovascular procedures. Aortic rupture. REBOA. Multiple trauma. Hemorrhage. Abdomen.

\section{Correspondencia:}

*Luis J. Fernández-López

E-mail: dr.fernandezlopez@gmail.com

DOI: 10.24875/RMA.21000022
Disponible en internet: 23-06-2021 Rev Mex Angiol. 2021;49(2):67-70 www.RMAngiologia.com 0377-4740/@ 2021 Sociedad Mexicana de Angiología y Cirugía Vascular y Endovascular, A.C. Publicado por Permanyer. Este es un artículo open access bajo la licencia CC BY-NC-ND license (http://creativecommons.org/licenses/by-nc-nd/4.0/). 


\section{Introducción}

La oclusión endovascular con balón de la aorta para reanimación (REBOA, resuscitative endovascular balloon occlusion of the aorta) es un procedimiento endovascular que consiste en insuflar un balón distensible en la aorta a través de un acceso arterial femoral con el objetivo de mejorar la perfusión de órganos vitales. Aunque no es un procedimiento nuevo, en los últimos años su uso ha adquirido relevancia e interés crecientes; se utiliza en el medio militar y en traumatismos civiles. La base teórica es similar a la de la toracotomía de reanimación (TR) con pinzamiento aórtico o masaje cardíaco abierto, pero con menor invasividad y morbilidad. Se ha observado que la fracción total de compresión cardíaca (TCCF) y la presión parcial de $\mathrm{CO}_{2}$ al final de la espiración (EtCO2) son más altas en pacientes que reciben REBOA en comparación con TR; esto representa mayor eficiencia del masaje cardíaco con REBOA ${ }^{1}$. Se considera que mejora la perfusión miocárdica durante el masaje cardíaco, aunque no hay evidencia definitiva de ello. Todo lo anterior es aplicable a otros escenarios, como hemorragia masiva obstétrica, digestiva, ruptura de aneurisma aórtico abdominal, entre otros, en los cuales el cirujano o el personal de emergencias entrenado pueden colocar el balón de manera segura en un minuto².

El ER-REBOA ${ }^{\circledR}$ es un catéter con dos luces, una para insuflar el globo y la otra para medir la presión proximal y obtener mediciones de presión; es curvo en la punta, con marcas en centímetros y se halla dentro de una vaina que debe removerse al introducirlo.

\section{Técnica de colocación}

Con ayuda del ultrasonido se coloca un introductor $7 \mathrm{Fr}$ en la arteria femoral común en sentido retrógrado y a continuación se introduce el catéter ER-REBOA ${ }^{\circledR}$ sin guía; de forma previa se mide la distancia del introductor al sitio donde el balón se insuflará, el balón se insufla con solución salina y medio de contraste con una jeringa de $20 \mathrm{ml}$ o $30 \mathrm{ml}$ y se corrobora el sitio del balón insuflado mediante rayos $X$.

La aorta se divide en tres zonas: zona 1 (aorta torácica) para hemorragia por abajo del diafragma (una contraindicación relativa es la lesión vascular por arriba del diafragma); zona 2 (aorta abdominal por debajo del diafragma y arriba de las arterias renales), en la cual está contraindicado insuflar por la presencia de arterias viscerales; y zona 3 (por debajo de las arterias renales) para hemorragia pélvica o de extremidad muy proximal y cuya contraindicación relativa es la lesión o hemorragia grave intraabdominal.

\section{Indicaciones para la colocación de REBOA}

1. Traumatismo: choque hemorrágico (fractura de cadera, hematoma retroperitoneal), paro cardíaco por choque hemorrágico o choque neurogénico e inestabilidad hemodinámica debida a episodios cardíacos ${ }^{3}$.

2. Sin traumatismo: choque hemorrágico (ginecoobstétrico, ortopédico, oncológico, digestivo, aneurisma abdominal roto) y paro cardíaco no hemorrágico, entre otros. El cirujano que atiende un choque grave puede usar REBOA en la placenta adherente, hemorragia posparto, hemorragia del tubo digestivo y casos diversos seleccionados de hemorragia intraabdominal no traumática ${ }^{4-6}$.

Brenner, et al. notificaron 60 casos de REBOA en tres centros de traumatismo, con $43 \%$ de sobrevida global (19\% en paro y $69 \%$ en hipotensión resistente); hasta $67.7 \%$ de los pacientes en paro regresaron a la circulación espontánea (ROSC), con $9.5 \%$ de sobrevida en paro por mecanismo contuso y $40 \%$ en paro por traumatismo penetrante ${ }^{7}$. En otro estudio de 285 pacientes, Brenner, et al. concluyeron que la REBOA confiere un beneficio en la sobrevida en comparación con TR, en particular en pacientes que no requieren $\mathrm{RCP}$, pero se necesitan más estudios ${ }^{8}$. DuBose, et al. señalaron que el introductor $7 \mathrm{Fr}$ (vs. $12 \mathrm{Fr}$ ) eleva la probabilidad de sobrevivir 24 horas y con cuatro veces menos probabilidad de embolismo distal ${ }^{9}$.

El uso de REBOA en el paro traumático hemorrágico es controversial y depende del escenario, se prolonga lo inevitable en algunos casos y se requieren recursos completos en quirófano, unidad de terapia intensiva y personal entrenado. Beldowicz, et al. comunicaron los primeros casos de traslado interhospitalario con la REBOA ${ }^{10}$. En casos seleccionados deben considerarse la donación de órganos y los factores emocionales de los familiares; si se decide usar hay que descartar hemorragia grave intratorácica y taponamiento cardíaco (con USG, cánula de tórax y radiografía); siempre se insuflará en la zona 1 cualquiera que sea la fuente de la hemorragia ${ }^{11,12}$.

Manzano-Núñez, et al. en el primer metaanálisis ( $n=1,276$ ), si bien en su mayor parte con datos observacionales, concluyeron que la REBOA tiene un efecto positivo en la mortalidad en pacientes con hemorragia de torso no comprimible, pero se requiere más investigación ${ }^{13}$. Bulger, et al., en un consenso de 2019, recomiendan no usar en la zona 1 si los pacientes no pueden llevarse pronto a quirófano para controlar el sangrado en los siguientes 15 minutos; el pinzamiento total mayor de 30 minutos se relaciona con aumento de complicaciones isquémicas y riesgo de muerte; el 


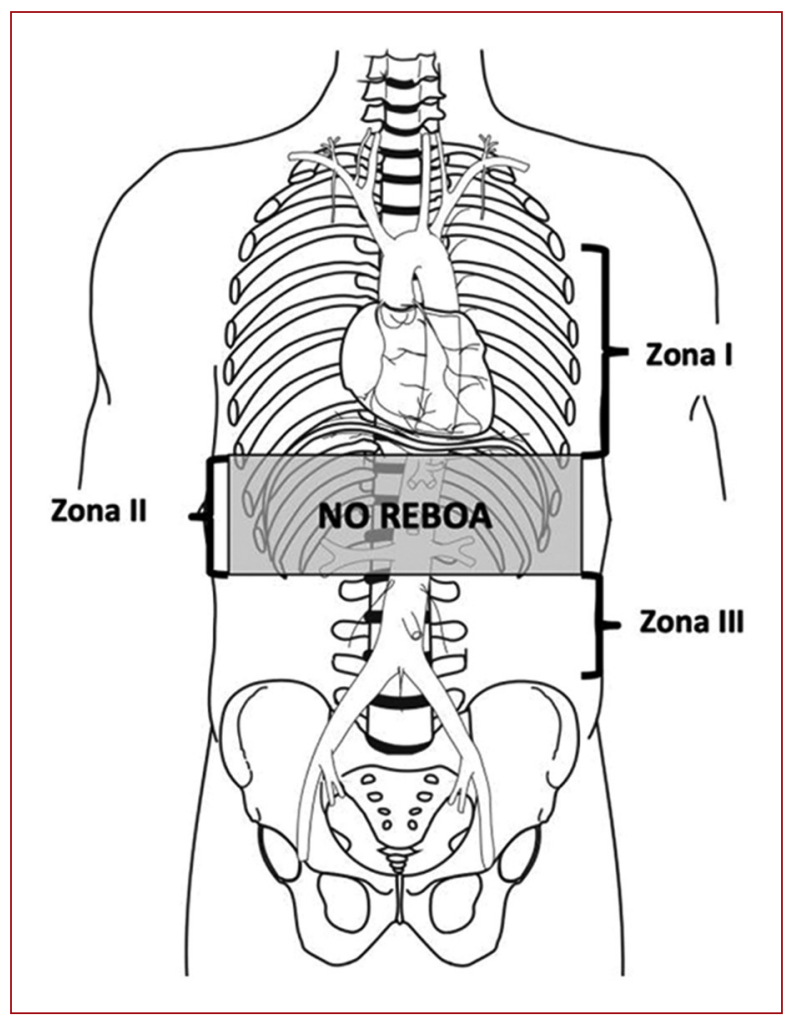

Figura 1. Zonas de la Aorta donde se puede inflar el dispositivo REBOA.

tiempo de oclusión máximo aceptable en la zona 3 se desconoce, pero el consenso recomienda enfocarse en menos de 30 minutos y no más de 60 ; todo lo anterior se basa en datos no publicados y consensos de opinión extrapolados de oclusión aórtica no traumática ${ }^{14,15}$.

Nothern, et al. informaron avances en cirugía austera realizada por fuerzas médicas para operaciones especiales en combates recientes con uso de REBOA ${ }^{16}$. Más información puede consultarse en las guías de práctica clínica para REBOA y en cuidados tácticos de víctimas de combate (TCCC, tactical combat casualty care), aunque estas no sustituyen al criterio clínico (Fig. 1) ${ }^{17}$.

\section{Conclusión}

La aplicación de la REBOA debe determinarse en cada escenario basado en el entrenamiento, experiencia, recursos locales y tiempos de evacuación. No hay evidencia clínica de niveles 1 o 2 que demuestre que la REBOA es superior a los métodos comunes de control de hemorragia (son posibles complicaciones la amputación o muerte); sin embargo, la REBOA puede ser un procedimiento que salve vidas en indicaciones y casos selecionados ${ }^{18,19}$.

\section{Agradecimientos}

Curso BEST para instructores en Cali, Colombia, 2019.

\section{Financiamiento}

La presente investigación no ha recibido ninguna beca específica de agencias de los sectores públicos o privados y sin ánimo de lucro.

\section{Conflicto de intereses}

Los autores declaran no tener conflicto de intereses.

\section{Responsabilidades éticas}

Protección de personas y animales. Los autores declaran que para esta investigación no se han realizado experimentos en seres humanos ni en animales.

Confidencialidad de los datos. Los autores declaran que en este artículo no aparecen datos de pacientes.

Derecho a la privacidad y consentimiento informado. Los autores declaran que en este artículo no aparecen datos de pacientes.

\section{Bibliografía}

1. Paradis NA, Martin GB, Rivers EP, Goetting MG, Appleton TJ, Feingold M, et al. Coronary perfusion pressure and the return of spontaneous circulation in human cardiopulmonary resuscitation. JAMA. 1990; 23,263(8):1106-13.

2. Bekdache O, Pardis T, Shen HBY, Elbahrawy A, Grushka J, Deckelbaum D, et al. Resuscitative endovascular balloon occlusion of the aorta (REBOA): indications: advantages and challenges of implementation in traumatic non-compressible torso hemorrhage. Trauma Surg Acute Care Open. 2019;4:e000262.

3. Tran NLT, Brasel JK, Karmy-Jones R, Rowell S, Schreiber AM, Shatz VD, et al. Western trauma association critical decisions in trauma: management of pelvic fracture with hemodynamic instability-2016 updates. Journal of Trauma and Acute Care Surgery. 2016;81(6):1171-1174.

4. Manzano-Núñez NR, Escobar VMF, Naranjo MP, Rodríguez F, Casallas JD, et al. Expanding the field of acute care surgery: a systematic review of the use of resuscitative endovascular balloon occlusion of the aorta (REBOA) in cases of morbidly adherent placenta. Eur J Trauma Emerg Surg. 2018;44(4):519-526.

5. Stensaeth HK, Sovik E, Haig YNI, Skomedal E, Jorgensen A. Fluoroscopy-free resuscitative endovascular balloon occlusion of the aorta (REBOA) for controlling life threatening postpartum hemorrhage. PLOS ONE. 2017;12(3):e0174520.

6. Hoehn RM, Hansraj ZN, Pasley MA, Brenner M, Cox RS, Pasley DJ, et al. Resuscitative endovascular balloon occlusion of the aorta for nontraumatic intra-abdominal hemorrhage. Eur J Trauma Emerg Surg. 2019;45:713.

7. Brenner M, Moore L, Teeter W, Hu P, Yang S, Wasicek P, et al. Exclusive clinical experience with a lower profile device for resuscitative endovascular balloon occlusion of the aorta (REBOA). The American Journal of Surgery. 2019;217(6):1126-1129. 
8. Brenner M, Inaba K, Aiolfi A, Dubose J, Fabian T, Bee T, et al Resuscitative endovascular balloon occlusion of the aorta and resuscitative thoracotomy in select patients with hemorrhagic shock: early results from the American Association for the Surgery of Trauma's Aortic Occlusion in Resuscitation for Trauma and Acute Care Surgery Registry. Journal of the American College of Surgeons. 2018;226(5):730-740.

9. DuBose JJ, Morrison J, Brenner M, Moore L, Holcomb JB, Inaba K, et al. Comparison of 7 and 11-12 French access for REBOA: results from the AAST aortic occlusion for resuscitation in trauma an acute care surgery (AORTA) Registry. JEVTM. 2019;(3)1:15-21

10. Beldowicz BC, Salcedo ES, Galante JM. Resuscitative endovascular balloon occlusion of the aorta in inter-hospital transfers: two case reports. JEVTM. 2018;(2)1:44-48

11. Brenner M, Hoehn M, Pasley J, Dubose J, Stein D, Scalea T. Basic endovascular skills for trauma course: bridging the gap between endovascular techniques and the acute care surgeon. Journal of Trauma and Acute Care Surgery. 2014;77(2):286-291

12. Brenner M, Moore L, Teeter W, Hu P, Yang S, Wasicek P, et al. Exclusive clinical experience with a lower profile device for resuscitative endovascular balloon occlusion of the aorta (REBOA). The American Journal of Surgery. 2018;217(6):1126-1129.

13. Manzano-Núñez R, Naranjo MP, Foianini E, Ferrada P, Rincon E, García-Perdomo $\mathrm{AH}$, et al. A meta-analysis of resuscitative endovascula balloon occlusion of the aorta (REBOA) or open aortic cross-clamping by resuscitative thoracotomy in non-compressible torso hemorrhage patients. World Journal of Emergency Surgery. 2017;12:30.
14. Bulger ME, Perina GD, Qasim Z, Beldowicz B, Brenner M, Guyette F, et al. Clinical use of resuscitative endovascular balloon occlusion of the aorta (REBOA) in civilian trauma systems in the USA, 2019: a joint statement from the American College of Surgeons Committee on Trauma, the American College of Emergency Physicians, the National Association of Emergency Medical Services Physicians and the National Association of Emergency Medical Technicians. Trauma Surgery \& Acute Care Open. 2019;4:e000376.

15. Romagnoli A, Teeter W, Pasley J, Hu P, Hoehn M, Stein D, et al. Time to aortic occlusion: It's all about access. J Trauma Acute Care Surg. 2017;83(6):1161-1164.

16. Northern DM, Manley JD, Lyon R, Barber D, Mitchell BJ, Filak KJ, et al. Recent advances in austere combat surgery: use of aortic balloon occlusion as well as blood challenges by special operations medical forces in recent combat operations. J Trauma Acute Care Surg. 2018; 85(2):98-103

17. Pasley JM, Cannon J, Glaser J, Polk T, Morrison J, Brocker J, et al. Resuscitative endovascular balloon occlusion of the aorta (REBOA) for hemorrhagic shock (CPG ID: 38). Joint Trauma System Clinical Practice Guideline (JTS CPG) 2017.

18. Russo MR, Neff PL, Lamb MC, Cannon WJ, Galante MJ, Clemente FN, et al. Partial resuscitative endovascular balloon occlusion of the aorta in swine model of hemorrhagic shock. Journal of the American College of Surgeons. 2016;223(2):359-368.

19. https://www.facs.org/quality-programs/traumatismo/education/best 